Editorial

For reprint orders, please contact: reprints@futuremedicine.com

\title{
Image-guided radiation therapy: looking beyond what we currently see
}

\author{
Caroline Chung ${ }^{*}, 1$ \& Kristy Brock ${ }^{2}$ \\ ${ }^{1}$ Department of Radiation Oncology/Diagnostic Imaging, University of Texas MD Anderson Cancer Center, 1515 Holcombe Blvd, \\ Houston, TX 77030, USA \\ ${ }^{2}$ Imaging Physics/Radiation Oncology, University of Texas MD Anderson Cancer Center, 1515 Holcombe Blvd, Houston, TX, 77030, \\ USA \\ * Author for correspondence: cchung3@mdanderson.org
}

First draft submitted: 30 June 2017; Accepted for publication: 10 July 2017; Published online: 10 November 2017

Keywords: adaptive $\bullet$ image-guided $\bullet$ radiotherapy/radiation therapy

Radiotherapy has evolved rapidly over several decades as the field has embraced advances in technology including digital imaging, accelerated computing and engineering solutions that have introduced increasingly sophisticated and efficient radiation delivery platforms. These advances have equipped us with the technology required to deliver increasingly conformal radiation treatments with greater precision that ever before. As a local therapy that relies on accurate targeting to achieve its therapeutic goals, these technical advances have also motivated a growing role for imaging in the radiotherapy workflow in order to ensure precise positioning and targeting.

The term image-guided radiation therapy has largely been accepted as the use of integrated imaging at the time of treatment to improve the precision of radiation delivery [1,2]. A range of imaging approaches are used today for in-room guidance of radiotherapy including planar imaging, volumetric imaging, cine imaging, fiducial marker and surface tracking. Various combinations of immobilization and image-guidance methodologies used at differing frequencies may be used to achieve the required treatment precision for a range of treatment techniques including 3D conformal radiotherapy, intensity-modulated radiotherapy, volumetric arc therapy or stereotactic radiosurgery/radiotherapy. The incorporation of image guidance in this context has largely focused on assuring consistent positioning of the patient and tumor. These efforts have been reported to improve clinical outcomes including increased tumor control and reduced normal tissue toxicity for various tumor sites [3-6].

The current approach for radiotherapy is based on dedicated effort by a multidisciplinary team to generate an optimized radiation plan prior to starting radiotherapy through the following processes: careful definition of the target volume and normal tissue structures; determination of dosimetry that ensures coverage of the target volumes while limiting dose to critical structures; quality and safety checks to ensure dose calculation accuracy. The radiotherapy treatment plan is based on one or more sets of images that may have been acquired immediately prior to the planning process or, in some cases, weeks prior to the planning process. This approach does not account for changes in the tumor or surrounding tissues from the time of image acquisition to the start of radiotherapy or any changes that may occur during the course of radiotherapy.

Clinical data have demonstrated that tumor and anatomical changes can occur during a course of radiotherapy in a number of tumor sites, including head and neck and cervical cancers $[7,8]$. When the original radiation plan is delivered under conditions of changing tumor volume and shifting organs at risk, the dose distribution across these structures can change significantly, resulting in over dosage to organs at risk and under dosage to tumor [9,10]. This evidence supports the use of adaptive radiotherapy approaches and incorporation of repeated imaging during a course of radiotherapy. However, the broad implementation of these approaches has been challenged by limited resources to acquire images enabling sufficient appreciation of soft tissue changes that can occur during radiotherapy and to generate adaptive radiation plans in a timely manner. Additionally, minimal investigation with serial imaging has been done for some tumors and normal tissues, which have been assumed to experience minimal change during a course of radiotherapy. However, the introduction of integrated magnetic resonance (MR)-linac systems has equipped us to evaluate tissue and tumor changes with serial imaging during radiotherapy across a broad range of clinical settings [11]. In parallel, newer treatment planning systems and approaches that provide inverse planning

Future Medicine 
solutions with rapid dose calculations have brought forth the possibility of real-time adaptive conformal radiotherapy approaches [12]. Therefore, we are now equipped technically to look beyond generating a single radiation plan to be delivered through the entire course of radiotherapy, but rather aim to achieve a specified cumulative radiation dose to be delivered to specific structures that will be reimaged and re-evaluated throughout a course of treatment.

Whether we are generating a single treatment plan at the start of radiotherapy or multiple iterations of adaptive radiotherapy plans over the course of treatment, the inverse planning approach relies heavily on accurate imaging data, target definition and normal tissue volumes. Avoiding or minimizing image distortion and artifacts is an essential step for high-quality image-guided radiotherapy [13,14]. Spatial fidelity is a particular challenge for MRI as multiple factors can introduce distortion both at the system and patient levels. However, approaches to minimize spatial distortion have not been a priority for MR utilization in the diagnostic setting. As the use of MR in radiotherapy is increasing, there are growing efforts to implement tools and processes for quality assurance checks that ensures high spatial fidelity in the MR images acquired for radiation treatment planning and guidance [15]. Within the American Association of Physicists in Medicine, Task Group No. 117 has started to evaluate the 'Use of MRI in Treatment Planning and Stereotactic Procedures - Spatial Accuracy and Quality Control Procedures' [16] and with increasing use of MR in radiotherapy, including MR-guided radiotherapy, there is growing need for standard quality practices to be developed.

Even with high-quality imaging, variability in target and normal tissue definition can result in wide variability in radiation treatment delivery, particularly for treatments that have higher conformality and precision such as radiosurgery [17-19]. Challenges in defining the gross tumor margin and clinical target margin for radiation planning have been a growing challenge in Radiation Oncology with increasing importance with the advent of inverse planning and increasingly conformal treatments, such as radiosurgery approaches. There have been a number of consensus-building efforts to increase the consistency of contours [20-23] and standardization of contouring practices can help us evaluate outcomes following a consensus approach. However, our ultimate goal is to define the pathological tumor margin on imaging so that we can target the gross disease that is present. The introduction of whole mount digital pathology has enabled direct correlation between pathology and imaging, and this equips us to pursue studies that get us closer to this ultimate goal by interrogating the imaging features associated with the pathological tumor margins. These same approaches can be used to explore other advanced biological, metabolic or molecular imaging features and the underlying biological characteristics that are present in the tumor and the tumor microenvironment. Noninvasive targeting of the pathological tumor margin and evaluation of biological characteristics in the whole tumor as well as subregions of tumor open up great therapeutic opportunities for radiation oncology.

As image guidance may be defined more broadly as any adjustments in treatment based on imaging findings, image-guided or image-directed radiation therapy may encompass determining the optimal temporal delivery of radiation therapy in addition to ensuring spatial precision in treatment delivery. Cancer treatment is increasingly multimodal and optimized coordination of multimodal therapy has the potential to improve outcomes. There is a promising role for imaging to help guide optimal sequencing and timing of multimodal treatment. With the ability to use imaging to evaluate the underlying biological changes, there is potential to measure early biological changes and explore optimal windows of time to expose the tumor to radiation to gain the maximal effect from radiation treatment in combination with various systemic therapies, such as immunotherapy.

Rapid advances in digital imaging, data processing and computing power has brought image-guided radiotherapy from fiction to reality and we are now better equipped than ever to look beyond what we can currently see. With the end goal of improving the therapeutic ratio by increase in tumor control and cancer outcomes while reducing treatment-related toxicity in order to preserve quality of life, our mission should be to collaboratively exploit the technology in order to generate agile, personalized, image-directed radiotherapy approaches for our patients.

Financial \& competing interests disclosure

The authors have no relevant affiliations or financial involvement with any organization or entity with a financial interest in or financial conflict with the subject matter or materials discussed in the manuscript. This includes employment, consultancies, honoraria, stock ownership or options, expert testimony, grants or patents received or pending, or royalties.

No writing assistance was utilized in the production of this manuscript. 


\section{References}

1. Verellen D, De Ridder M, Linthout N, Tournel K, Soete G, Storme G. Innovations in image-guided radiotherapy. Nat. Rev. Cancer 7(12), 949-960 (2007).

2. Jaffray DA. Image-guided radiotherapy: from current concept to future perspectives. Nat. Rev. Clin. Oncol. 9(12), 688-699 (2012).

3. Gill S, Thomas J, Fox C et al. Acute toxicity in prostate cancer patients treated with and without image-guided radiotherapy. Radiat. Oncol. 6, 145 (2011).

4. Zelefsky MJ, Kollmeier M, Cox B et al. Improved clinical outcomes with high-dose image guided radiotherapy compared with non-IGRT for the treatment of clinically localized prostate cancer. Int. J. Radiat. Oncol. Biol. Phys. 84(1), 125-129 (2012).

5. Ward MC, Ross RB, Koyfman SA et al. Modern image-guided intensity-modulated radiotherapy for oropharynx cancer and severe late toxic effects: implications for clinical trial design. JAMA Otolaryngol. Head Neck Surg. 142(12), 1164-1170 (2016).

6. Bujold A, Craig T, Jaffray D, Dawson LA. Image-guided radiotherapy: has it influenced patient outcomes? Semin. Radiat. Oncol. 22(1), 50-61 (2012).

7. Hamming-Vrieze O, Van Kranen SR, Heemsbergen WD et al. Analysis of GTV reduction during radiotherapy for oropharyngeal cancer: implications for adaptive radiotherapy. Radiother. Oncol. 122(2), 224-228 (2017).

8. Jadon R, Pembroke CA, Hanna CL et al. A systematic review of organ motion and image-guided strategies in external beam radiotherapy for cervical cancer. Clin. Oncol. (R. Coll. Radiol.) 26(4), 185-196 (2014).

9. Barker JL Jr, Garden AS, Ang KK et al. Quantification of volumetric and geometric changes occurring during fractionated radiotherapy for head-and-neck cancer using an integrated CT/linear accelerator system. Int. J. Radiat. Oncol. Biol. Phys. 59(4), 960-970 (2004).

10. Castadot P, Lee JA, Geets X, Gregoire V. Adaptive radiotherapy of head and neck cancer. Semin. Radiat. Oncol. 20(2), 84-93 (2010).

11. Glitzner M, Crijns SP, De Senneville BD et al. On-line MR imaging for dose validation of abdominal radiotherapy. Phys. Med. Biol. 60(22), 8869-8883 (2015).

12. Kontaxis C, Bol GH, Lagendijk JJ, Raaymakers BW. Towards adaptive IMRT sequencing for the MR-linac. Phys. Med. Biol. 60(6), 2493-2509 (2015).

13. Mao W, Speiser M, Medin P, Papiez L, Solberg T, Xing L. Initial application of a geometric QA tool for integrated MV and $\mathrm{kV}$ imaging systems on three image guided radiotherapy systems. Med. Phys. 38(5), 2335-2341 (2011).

14. Chiu TD, Yan Y, Foster R, Mao W. Long-term evaluation and cross-checking of two geometric calibrations of kV and MV imaging systems for Linacs. J. Appl. Clin. Med. Phys. 16(4), 306-310 (2015).

15. Crijns SP, Bakker CJ, Seevinck PR, De Leeuw H, Lagendijk JJ, Raaymakers BW. Towards inherently distortion-free MR images for image-guided radiotherapy on an MRI accelerator. Phys. Med. Biol. 57(5), 1349-1358 (2012).

16. AAPM. https://www.aapm.org/org/structure/?committee_code=TG117

17. Sandstrom H, Chung C, Jokura H, Torrens M, Jaffray D, Toma-Dasu I. Assessment of organs-at-risk contouring practices in radiosurgery institutions around the world - the first initiative of the OAR Standardization Working Group. Radiother. Oncol. 121(2), 180-186 (2016).

18. Stanley J, Dunscombe P, Lau H et al. The effect of contouring variability on dosimetric parameters for brain metastases treated with stereotactic radiosurgery. Int. J. Radiat. Oncol. Biol. Phys. 87(5), 924-931 (2013).

19. Li XA, Tai A, Arthur DW et al. Variability of target and normal structure delineation for breast cancer radiotherapy: an RTOG multi-institutional and multiobserver study. Int. J. Radiat. Oncol. Biol. Phys. 73(3), 944-951 (2009).

20. Redmond KJ, Robertson S, Lo SS et al. Consensus contouring guidelines for postoperative stereotactic body radiation therapy for metastatic solid tumor malignancies to the spine. Int. J. Radiat. Oncol. Biol. Phys. 97(1), 64-74 (2017).

21. Gaffney DK, King B, Viswanathan AN et al. Consensus recommendations for radiation therapy contouring and treatment of vulvar carcinoma. Int. J. Radiat. Oncol. Biol. Phys. 95(4), 1191-1200 (2016).

22. Niyazi M, Brada M, Chalmers AJ et al. ESTRO-ACROP guideline "target delineation of glioblastomas". Radiother. Oncol. 118(1), 35-42 (2016).

23. Harris VA, Staffurth J, Naismith O et al. Consensus guidelines and contouring atlas for pelvic node delineation in prostate and pelvic node intensity modulated radiation therapy. Int. J. Radiat. Oncol. Biol. Phys. 92(4), 874-883 (2015). 
